\title{
Bericht über die Arbeit der Menschenrechtskommission in Mexiko - Dritter Halbjahresbericht der Comisión Nacional de Derechos Humanos
}

\author{
Von Hans-Rudolf Horn
}

Am 5. Juni 1990 wurde durch Dekret des mexikanischen Präsidenten Carlos Salinas de Gortari die Nationale Menschenrechtskommission ins Leben gerufen. Ihr Präsident ist Jorge Carpizo, der sich als Verfassungsrechtler, Direktor des Rechtswissenschaftlichen Forschungsinstituts der Autonomen Nationaluniversität Mexiko und dann als deren Rektor international einen Namen gemacht hat. Der Dritte Halbjahresbericht, der am 4. Dezember 1991 vorgelegt wurde, umfaßt drei Bände mit insgesamt 1.685 Druckseiten, die nicht nur Einzelfälle, sondern auch systematisch erarbeitete Vorschläge beispielsweise zur Verbesserung des Strafvollzugs zum Gegenstand haben. Eine Zusammenfassung ist in übersichtlicher Form auf 141 Seiten in einer Auflage von 5.000 Exemplaren veröffentlicht worden.

Von Juni bis Dezember 1991 wurden 2.485 neue Beschwerden erhoben und 1.892 abgeschlossen. Durchschnittlich gingen jeden Kalendertag mehr als 13 Beschwerden ein, erledigt wurden durchschnittlich über 10 am Tag. Im letzten Halbjahr wurden mehr Beschwerden erledigt als im gesamten ersten Jahr des Bestehens der Kommission. Im Einvernehmen mit den betroffenen Behörden wurden ungefähr $30 \%$ mehr Fälle gelöst als im vorangegangenen Halbjahr. Unterstützt wurde dieses kooperative Verfahren am meisten durch die Generalbundesanwaltschaft und die Regierungen der Bundesstaaten México, Yucatán, Hidalgo, Oaxaca und Nuevo León.

Die Beschuldigungen, die Untersuchungen ausgelöst haben, beziehen sich - geordnet nach ihrer Häufigkeit - auf willkürliche Festnahmen, falsche Anklagen, Folter, Amtsmißbrauch, Rechtsverweigerung und Rechtsverzögerung. In den beiden ersten Halbjahresberichten standen die Fälle, in denen der Foltervorwurf erhoben wurde (180), mit 13,4\% bzw. 266 mit $13,9 \%$ noch an erster Stelle, im dritten Halbjahresbericht sind sie mit 156 Fällen $(6,2 \%)$ an die dritte Stelle gerückt.

Die drei einzigen Fälle aus dem ersten Halbjahresbericht, bei denen die Empfehlungen der Menschenrechtskommission noch nicht verwirklicht sind, entbehren nicht einer besonderen Dramatik. So konnten die mutmaßlichen Urheber des Mordes an einem Bezirksrichter im Staate Guerrero fliehen, weil der zuständige Haftrichter die erforderlichen Sicherungsmaßnahmen nicht angeordnet hatte. Der erneute Haftbefehl konnte noch nicht vollstreckt 
werden. Der Kommission liegen auch noch keine Meldungen darüber vor, warum die unberechtigte Verhaftung von Indios im Staate Veracruz noch nicht geahndet wurde. Mangelnde Bereitschaft bestimmter Stellen, mit der Kommission zusammenzuarbeiten, wird beispielsweise im Falle des Gouverneurs von Tamaulipas ausdrücklich angeprangert. Hervorgehoben wird aber, daß im Berichtszeitraum in $94 \%$ der Fälle eine angemessene Antwort eingegangen ist, während diese Quote im vorangegangenen Halbjahr noch bei $62 \%$ lag.

Ein besonders bemerkenswerter Fall aus dem zweiten Halbjahresbericht vom 5. Juni 1991 konnte inzwischen aufgeklärt werden. Opfer eines Mordkomplotts war Frau Dr. Norma Corona, die im Staate Sinaloa den Drogenhandel erfolgreich bekämpft hatte. Die außerordentliche Bedeutung des Wirkens der mexikanischen Menschenrechtskommission läßt sich daraus ersehen, daß 139 öffentlich-rechtlich Bedienstete belangt worden sind, davon 93 des Bundes, 36 der Einzelstaaten und 10 der Gemeinden. Von diesen wurden 37 ihres Amtes enthoben, 36 vom Dienst suspendiert, gegen 64 wurde Anklage erhoben und zwei erhielten einen Verweis.

Die Kommission wird sich nicht auf ihren Lorbeeren ausruhen. Insbesondere kommt es ihr darauf an, ihre gezielte Informations- und Reformpolitik fortzusetzen. Im Verlauf des bereits erwähnten Programms zur Verbesserung des Strafvollzugs wurden bereits 93 Strafgefangene - daneben fünf weitere aus gesundheitlichen Gründen - vorzeitig entlassen; ferner wurden weitere Schritte gegen die Korruption in Gefängnissen eingeleitet. Flankiert werden diese Maßnahmen durch spezielle Schulungsprogramme für das Gefängnispersonal und die Polizei mit 34 Kursen ("Programas de Capacitación"). Im Rahmen des Programms für Indio-Angelegenheiten wurden 75 Indios vorzeitig aus dem Gefängnis entlassen. Bei der Umsetzung des Programms für angeblich Verschwundene wurden 26 weitere Fälle aufgeklärt, deren Gesamtzahl auf 40 stieg.

Insgesamt 13 Kongresse, Seminare und Kolloquien über Menschenrechtsfragen wurden veranstaltet. Besonders hervorzuheben ist der Internationale Kongreß über die Erfahrungen mit dem Ombudsman in der Gegenwart. 421 Teilnehmer interessierten sich für die Erfahrungen von Ombudsmännern aus aller Welt, darunter denen aus Dänemark und Schweden und dem aus Spanien (dort "defensor del pueblo" genannt). Die enge Zusammenarbeit mit der Justiz und der Rechtswissenschaft wurde vor allem auch durch das iberoamerikanischspanische Symposion im Rechtswissenschaftlichen Forschungsinstitut der Autonomen Nationaluniversität Mexiko und das Forum zum Schutz der Menschenrechte in Tlaxcala zum Ausdruck gebracht.

Nicht weniger wichtig als internationale akademische Veranstaltungen sind die Bemühungen der mexikanischen Menschenrechtskommission, den Gedanken des Schutzes der Menschenrechte über die modernen Massenmedien an die heranzubringen, die es angeht. Jeden Sonntag erscheinen in der Tageszeitung "El Nacional" Informationen über die Tätig- 
keit der Kommission. Elementare Hinweise zu dem Einreichen einer Beschwerde werden seit dem 2. September 1991 über 40 Radiostationen der Hauptstadt und 265 Stationen in allen Staaten der Republik verbreitet.

Die Bemühungen um den Schutz der Menschenrechte erhalten ihre Bedeutung auch vor dem Hintergrund, daß in unseren Medien immer wieder Meldungen über Menschenrechtsverletzungen in Lateinamerika verbreitet werden. Mancher mag seine Vorurteile durch die Fälle, über die von der Kommission berichtet wird, bestätigt sehen und dabei aus dem Auge verlieren, daß Menschenrechtsverletzungen in den Ländern am schlimmsten sind, aus denen man nichts darüber hört, weil weder Journalisten noch Gefangenenhilfsorganisationen Zugang zu Informationen erhalten. Die Landkarten Mexikos und der übrigen lateinamerikanischen Länder haben in dieser Hinsicht immer weniger weiße Flecken. 